\title{
Cristalização da zeólita mordenita com adição de sementes em meio reacionais com diferentes razões $\mathrm{SiO}_{2} / \mathrm{Al}_{2} \mathrm{O}_{3}$.
}

\author{
Crystallization of mordenite zeolite with seed addition in reaction mixtures with different
} $\mathrm{SiO}_{2} / \mathrm{Al}_{2} \mathrm{O}_{3}$ ratios.

\author{
O. M. S. Cysneiros; B. J. B. Silva*; A. O. S. Silva; S. L. Alencar; R. B. Santos; P. \\ F. M. Soares; T. R. D. Mendonça; L. V. Sousa Júnior; J. R. Santos \\ Departamento de Engenharia Química/Laboratório de Sintese de Catalisadores, Universidade Federal de Alagoas, \\ CEP:57082-900, Maceió-Alagoas, Brasil
}

*brunojbarros@hotmail.com

(Recebido em 07 de janeiro de 2016; aceito em 10 de março de 2016)

\begin{abstract}
A síntese das amostras de mordenita foram realizadas através do método hidrotérmico, utilizando dois tipos diferentes de sementes de cristalização (mordenita e ferrierita), onde foram explorados a variação de alguns parâmetros de síntese, como a razão $\mathrm{SiO}_{2} / \mathrm{Al}_{2} \mathrm{O}_{3}=20,60$ e 100, e a razão $\mathrm{OH} / \mathrm{SiO}_{2}=0,2,0,3$ e 0,4 , em diferentes tempos de cristalização, à temperatura de $170{ }^{\circ} \mathrm{C}$. As amostras foram caracterizadas por difratometria de raios X (DRX), análises termogravimétricas (ATG), adsorção de nitrogênio a -196 ${ }^{\circ} \mathrm{C}$ (BET) e dessorção à temperatura programada de amônia $\left(\mathrm{DTP}-\mathrm{NH}_{3}\right)$. Constatou-se que a utilização da razão $\mathrm{OH} / \mathrm{SiO}_{2}=0,4$, em conjunto com as sementes de MOR e FER foram obtidas as amostras mais cristalinas, evidenciando o papel crucial do agente mineralizante $\mathrm{OH}^{-}$no meio.
\end{abstract}

Palavras-chave: síntese, zeólita, mordenita.

The synthesis of samples of mordenite were performed using the hydrothermal method using two different types of crystallization seed (mordenite and ferrierite), where they were exploited to change some parameters of the synthesis, such as $\mathrm{SiO}_{2} / \mathrm{Al}_{2} \mathrm{O}_{3}=20,60$ and 100 and the ratio $\mathrm{OH} / \mathrm{SiO}_{2}=0.2,0.3$ and 0.4 , at different times of crystallization, at a temperature of $170{ }^{\circ} \mathrm{C}$. The samples were characterized by X-ray diffraction (XRD), thermogravimetric analysis (TGA/DTA), nitrogen adsorption at $-196{ }^{\circ} \mathrm{C}(\mathrm{BET})$ and temperature programmed desorption of ammonia $\left(\mathrm{NH}_{3}-\mathrm{TPD}\right)$. It was found that the use of the ratio $\mathrm{OH} / \mathrm{SiO}_{2}=0.4$, together with the seeds of the most MOR and FER crystalline samples were obtained, demonstrating the critical role of mineralizing agent $\mathrm{OH}^{-}$in the middle.

Keywords: synthesis, zeolite, mordenite.

\section{INTRODUÇÃO}

A mordenita (MOR) é uma zeólita com composição química igual a $\mathrm{Na}_{8} \mathrm{Al}_{8} \mathrm{Si}_{40} \mathrm{O}_{96} \cdot \mathrm{nH}_{2} \mathrm{O}$ e possui cela unitária ortorrômbica com as seguintes dimensões: $\mathrm{a}=18,1 \AA$, $\mathrm{b}=20,5 \AA \mathrm{e} \mathrm{c}=7,5 \AA$ e simetria do grupo espacial $\mathrm{Cmcm}$, sua morfologia é caracterizada por cristais em forma de agulhas na direção do eixo c [1-2]. A mordenita possui uma relação $\mathrm{Si} / \mathrm{Al} \geq 5$, sendo formada por dois sistemas de canais de abertura elíptica que se interconectam através da combinação de anéis de cinco tetraedros, com 12 átomos de oxigênio medindo $6,5 \times 7,0 \AA$, unidos entre si por anéis de quatro tetraedros, com 8 átomos de oxigênio, com diâmetro de 2,6 x 5,7 A, formando cadeias que originam lâminas características da mordenita [3].

Devido a sua alta estabilidade térmica e acidez, a zeólita mordenita é utilizada como adsorvente na separação de gases ou misturas líquidas, tão como catalisador nos processos de hidrocraqueamento, hidroisomerização, alquilação, reforma catalítica, desparafinação e síntese de dimetilaminas [4-5]. Além disso, a mordenita foi recentemente aplicada como matriz de materiais de semicondutores, sensores químicos e materiais não-lineares [6].

Atualmente, existe um grande interesse na síntese da mordenita, especialmente com altas razões $\mathrm{SiO}_{2} / \mathrm{Al}_{2} \mathrm{O}_{3}$, por proporcionar uma alta estabilidade térmica da estrutura. Zeólitas com altas razões de sílica-alumina são geralmente preparadas pela remoção de alumínio da rede estrutural zeolítica,

$$
\text { 043101-1 }
$$


através de tratamento com vapor de água e/ou ácido [7]. Porém, uma zeólita que sofre o processo de desaluminação, geralmente, torna-se menos estável termicamente em comparação às zeólitas com altas razões $\mathrm{SiO}_{2} / \mathrm{Al}_{2} \mathrm{O}_{3}[8]$.

A zólita mordenita com altas razões $\mathrm{SiO}_{2} / \mathrm{Al}_{2} \mathrm{O}_{3}$, vem sendo sintetizada através da utilização de agentes orgânicos direcionadores de estrutura, como o hidróxido de benziltrimetilamônio, hidróxido de tetraetilamônio, hexametileneimina e o benzeno-1,2-diol [9-10], no entanto, estes compostos elevam significativamente o custo de produção dos materiais zeolíticos, podendo originar efluentes líquidos e gasosos tóxicos durante as etapas de separação e calcinação dos sólidos [11]. Já está estabelecido, desde 1960, que a adição de sementes de cristalização no gel inicial, acarreta no aumento da taxa de cristalização, encurtando-se o tempo requerido para a cristalização completa do material, permitindo o controle da contaminação por outras estruturas zeolíticas [12] e a eliminação, parcial ou total, do uso de compostos orgânicos empregados na síntese [13].

O presente estudo objetivou a avaliação da cristalização da zeólita mordenita, com uso de dois tipos diferentes de sementes de cristalização (MOR e FER), variando-se as razões $\mathrm{SiO}_{2} / \mathrm{Al}_{2} \mathrm{O}_{3}$ (SAR) e $\mathrm{OH} / \mathrm{SiO}_{2}$, em diferentes tempos de cristalização.

\section{MATERIAL E MÉTODOS}

\subsection{SÍNTESE DAS SEMENTES DE CRISTALIZAÇÃO}

Para a síntese da semente de MOR utilizou-se a seguinte composição molar $20 \mathrm{SiO}_{2}: 1,0 \mathrm{Al}_{2} \mathrm{O}_{3}$ : 7,0 $\mathrm{Na}_{2} \mathrm{O}: 3,0 \mathrm{H}_{2} \mathrm{SO}_{4}: 333,33 \mathrm{H}_{2} \mathrm{O}$, onde para obtenção do gel foram utilizados: silicato de sódio $\left(11,20 \% \mathrm{Na}_{2} \mathrm{O}, 31,50 \%\right.$ de $\mathrm{SiO}_{2}$ e $57,30 \%$ de $\mathrm{H}_{2} \mathrm{O} \% \mathrm{p} / \mathrm{p}$, Pernambuco Química), sulfato de alumínio hidratado (99\% p/p, Merck), ácido sulfúrico (98\% p/p, Aldrich). Posteriormente o gel foi transferido para um reator Parr de $1 \mathrm{~L}$, sob agitação de $300 \mathrm{rpm}$, e aquecido a $170{ }^{\circ} \mathrm{C}$ por 24 horas. Após a cristalização, o sólido resultante do processo de cristalização foi separado por centrifugação e seco em estufa a $120{ }^{\circ} \mathrm{C}$ por 12 horas.

Foi utilizada como semente de FER uma amostra comercial (CP914C), adquirida do fabricante de zeólitas Zeolyst Corporation.

\subsection{SÍNTESE DAS AMOSTRAS DE MORDENITA}

Foram sintetizadas amostras sem e com $10 \%$ em massa de sementes em relação a $\mathrm{SiO}_{2}$, com regentes similares aos utilizados na síntese da semente de MOR, variando-se as razões de $\mathrm{SiO}_{2} / \mathrm{Al}_{2} \mathrm{O}_{3}=20,60$ e 100 e $\mathrm{OH} / \mathrm{SiO}_{2}=0,2,0,3$ e 0,4. Após o preparo do gel, este foi transferido para vasos de teflon de $70 \mathrm{~mL}$, revestido com autoclaves de aço inoxidável e levados a estufa sob aquecimento a $170{ }^{\circ} \mathrm{C}$, por diversos tempos de cristalização, sem agitação do conteúdo da autoclave. Posteriormente, o sólido resultante do processo de cristalização foi separado por filtração à vácuo, e seco em estufa a $120^{\circ} \mathrm{C}$ por 12 horas.

\subsection{CARACTERIZAÇÔES}

\subsubsection{DIFRATOMETRIA DE RAIOS X (DRX)}

As amostras foram caracterizadas utilizando um difratômetro Shimadzu, modelo XRD-6000, com radiação $\mathrm{CuK} \alpha(\lambda=0,1542 \mathrm{~nm})$, filtro de $\mathrm{Ni}$, voltagem de $40 \mathrm{kV}$ e corrente de $30 \mathrm{~mA}$. A aquisição dos dados foi realizada no intervalo de $2 \theta$ entre 3 e $40^{\circ}$, com velocidade de varredura de goniômetro de $2^{\circ} \cdot \min ^{-1}$ e passo de $0,02^{\circ}$. 


\subsubsection{ANÁLISES TERMORGRAVIMETRICAS (ATG)}

As análises foram realizadas numa termobalança Shimadzu, modelo DTG-60H, onde foram utilizados cadinhos de alumina e massas de amostra de aproximadamente $10 \mathrm{mg}$. Foi utilizada uma razão de aquecimento $10{ }^{\circ} \mathrm{C} / \mathrm{min}$, na faixa de temperatura ambiente até $800{ }^{\circ} \mathrm{C}$, em atmosfera oxidante com vazão de $50 \mathrm{~mL} \cdot \mathrm{min}^{-1}$.

\subsubsection{ADSORÇÃO DE $\mathrm{N}_{2} \mathrm{~A}-196^{\circ} \mathrm{C}(\mathrm{BET})$}

As medidas de adsorção de nitrogênio foram realizadas num equipamento Micromeritics, modelo ASAP 2020 , a $-196{ }^{\circ} \mathrm{C}$ e anteriormente desgaseificadas por 12 horas, sob vácuo $(2 \mu \mathrm{m}$ de mercúrio) a $350{ }^{\circ} \mathrm{C}$, com o objetivo de remover qualquer espécie fisissorvida na superfície da amostra.

\subsubsection{DESSORÇÃO À TEMPERATURA PROGRAMADA DE AMÔNIA (DTP-NH3)}

Previamente, as amostras foram submetidas à troca iônica utilizando o uma solução de $1 \mathrm{M}$ de nitrato de amônio $\left(\mathrm{NH}_{4} \mathrm{NO}_{3}, 98 \% \mathrm{p} / \mathrm{p}\right)$. As amostras foram submetidas a um pré-tratamento a $500{ }^{\circ} \mathrm{C}$ em atmosfera de hélio com vazão de $30 \mathrm{~mL} \cdot \mathrm{min}^{-1}$. Em seguida, a temperatura foi reduzida a $100^{\circ} \mathrm{C}$ e a amostra submetida à corrente de amônia, para adsorção química, por 40 minutos, para assegurar completa saturação de todos os centros ácidos. A etapa final do processo de adsorção consiste na remoção das moléculas de $\mathrm{NH}_{3}$ fisissorvidas por 1 hora a $100{ }^{\circ} \mathrm{C}$, em vazão de hélio $30 \mathrm{~mL} \cdot \mathrm{min}^{-1}$. Os termogramas foram obtidos através do aquecimento de 100 a $800{ }^{\circ} \mathrm{C}$, com uma taxa de aquecimento de $10{ }^{\circ} \mathrm{C} \cdot \mathrm{min}^{-1}$ sob vazão de hélio de $30 \mathrm{~mL} \cdot \mathrm{min}^{-1}$.

\subsubsection{MICROSCOPIA ELETRÔNICA DE VARREDURA (MEV)}

As análises foram realizadas num equipamento Shimadzu, modelo SSX-550. As amostras foram suspensas numa pequena quantidade de acetona para melhorar a dispersão dos cristais, seguida da deposição de uma gota desta suspensão sobre uma fita de carbono aderida ao porta amostra. Para se obter uma melhor resolução das imagens uma fina camada de ouro foi depositada sobre a amostra, e que a mesma foi levada ao metalizador Sanyu Electron, modelo Quick Coater SC-701, com corrente durante o processo de metalização de $10 \mathrm{~mA}$ por 8 minutos.

\section{RESULTADOS E DISCUSSÃO}

\subsection{DIFRATOMETRIA DE RAIOS - X (DRX)}

As amostras sintetizadas sem sementes, razões $\mathrm{SiO}_{2} / \mathrm{Al}_{2} \mathrm{O}_{3}=20$ e $\mathrm{OH} / \mathrm{SiO}_{2}=0,2$ e 0,3 , não apresentaram uma evolução em sua cristalização, demonstrando sólidos completamente amorfos, mesmo com tempo relativamente longos, em torno de 72 horas. Já as amostras sintetizadas com sementes de FER, $\mathrm{SAR}=20$ e razão $\mathrm{OH} / \mathrm{SiO}_{2}=0,2$, o comportamento verificado foi a coexistência das fases mordenita e ferrierita em todos os períodos de cristalização. A Tabela 1 apresenta as séries de experimentos em que se obtiveram a fase mordenita isenta de contaminantes. 
Tabela 1: Dados das amostras com razão $\mathrm{SiO}_{2} / \mathrm{Al}_{2} \mathrm{O}_{3}=20$.

\begin{tabular}{lcccc}
\hline \multicolumn{1}{c}{ Amostra } & Razão OH/SiO $\mathbf{2}_{2}$ & $\begin{array}{c}\text { Tipo de } \\
\text { semente }\end{array}$ & $\begin{array}{c}\text { Tempo } \\
\text { (horas) }\end{array}$ & Cristalinidade (\%) \\
\hline Semente-MOR & 0,4 & --- & 24 & $100^{*}$ \\
M20B20SM-4H & 0,2 & MOR & 4 & 39 \\
M20B20SM-8H & 0,2 & MOR & 8 & 48 \\
M20B20SM-12H & 0,2 & MOR & 12 & 88 \\
M20B20SM-24H & 0,2 & MOR & 24 & 84 \\
M20B30SM-4H & 0,3 & MOR & 4 & 83 \\
M20B30SM-8H & 0,3 & MOR & 8 & 85 \\
M20B30SM-12H & 0,3 & MOR & 12 & 84 \\
M20B30SM-24H & 0,3 & MOR & 24 & 81 \\
M20B30SF-8H & 0,3 & FER & 8 & 83 \\
M20B30SF-12H & 0,3 & FER & 12 & 88 \\
M20B30SF-24H & 0,3 & FER & 24 & 36 \\
M20B40S0-24H & 0,4 & -- & 24 & 99 \\
M20B40S0-48H & 0,4 & --- & 48 & 88 \\
M20B40S0-72H & 0,4 & -- & 72 & 99 \\
M20B40S0-96H & 0,4 & --- & 96 & 103 \\
M20B40SM-2H & 0,4 & MOR & 2 & 105 \\
M20B40SM-8H & 0,4 & MOR & 8 & 99 \\
M20B40SM-12H & 0,4 & MOR & 12 & 103 \\
M20B40SM-24H & 0,4 & MOR & 24 & 105 \\
M20B40SF-8H & 0,4 & FER & 8 & 106 \\
M20B40SF-12H & 0,4 & FER & 12 & \\
M20B40SF-24H & 0,4 & FER & 24 & \\
\hline A & & & & 93 \\
\hline
\end{tabular}

* Amostra padrão

A Figura 1 apresenta os DRX das amostras mais cristalinas, sintetizadas com razão $\mathrm{SiO}_{2} / \mathrm{Al}_{2} \mathrm{O}_{3}$ $=20$ e razão $\mathrm{OH} / \mathrm{SiO}_{2}=0,4$. Observou-se que em meios reacionais mais alcalinos (razão $\mathrm{OH} / \mathrm{SiO}_{2}$ $=0,4$ ) foi possível a obtenção da fase mordenita sem a adição de sementes. Estes resultados evidenciaram a efetividade da adição de sementes de MOR e FER para a síntese de zeólita mordenita. Conforme indicado na Tabela 1, a adição de sementes de MOR possibilitou a obtenção de amostras com cristalinidade de $99 \%$ já a partir de 2 horas de aquecimento, enquanto que com adição das sementes de FER, somente após $8 \mathrm{~h}$ de síntese é que foi obtido o material completamente cristalino, indicando que as sementes de FER não nucleiam a formação da zeólita mordenita tão rapidamente quanto as sementes de MOR. Esta diferença de comportamento pode estar relacionada a forma como os dois tipos atuam na nucleação. Itabashi e colaboradores propõem que existe uma subunidade da zeólita mordenita, que é comum também para as zeólitas ferrierita e BEA. Esta subunidade formada por oito tetraedros $\mathrm{TO}_{4}$ seria o precursor necessário para a cristalização das zeólitas com este tipo de característica estrutural [14]. Com base neste princípio, a adição de sementes de FER, num meio reacional mais propicio a formação da zeólita mordenita, inicialmente provoca a nucleação e crescimento da própria fase ferrierita. Decorrido algum tempo, parte das sementes de FER se dissolve no meio e expõe subunidades estruturais comuns a mordenita, as quais começam a nuclear a fase MOR e, devido ao meio ser mais favorável à formação desta zeólita, ocorre um rápido crescimento dos seus cristais, concomitantemente com a dissolução dos cristais da fase FER que foram adicionados e formados na fase inicial da síntese. 


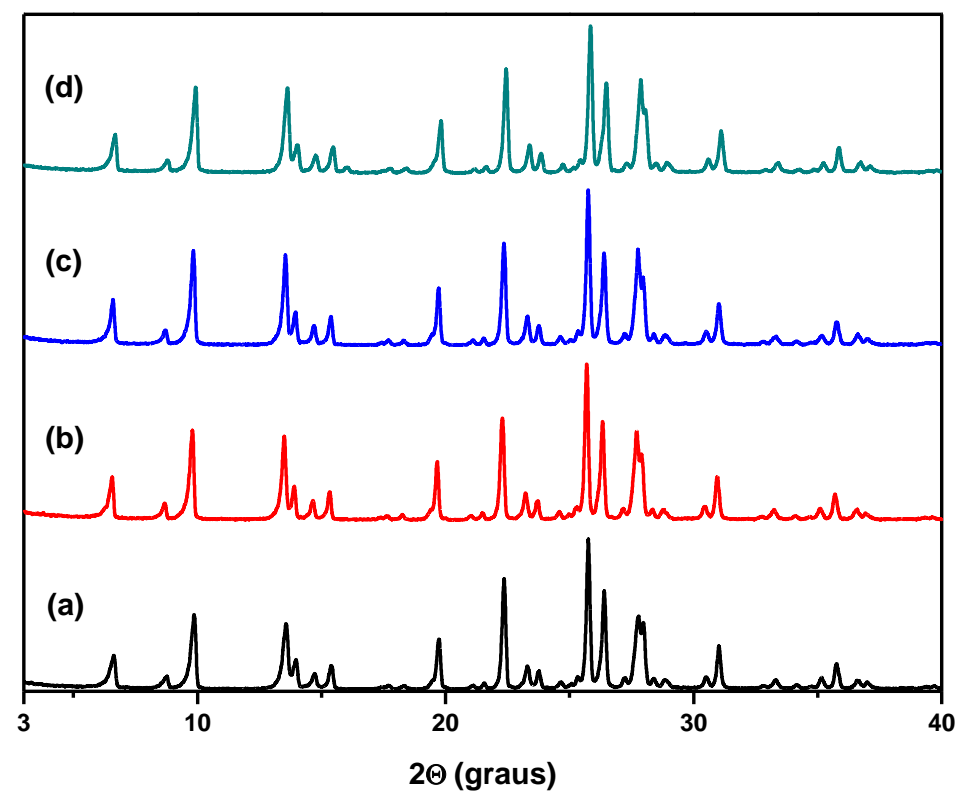

Figura 1:Difratograma das amostras mais cristalinas com razão $\mathrm{SiO}_{2} / \mathrm{Al}_{2} \mathrm{O}_{3}=20$. (a) Semente-MOR; (b) M20B40-48H; (c) M20B40SM-12H; (d) M20B40SF-24H.

Nas amostras com razão $\mathrm{SiO}_{2} / \mathrm{Al}_{2} \mathrm{O}_{3}=60$ e sem adição de sementes, não foi observada a formação de fases cristalinas no decorrer do processo de aquecimento, obtendo-se sólidos amorfos em todos os tempos de cristalização (que foi de até 72 horas). Em contrapartida, as amostras sintetizadas utilizando as sementes de FER, apresentaram a coexistência das fases mordenita e ferrierita, conforme mostrado na Figura 2.

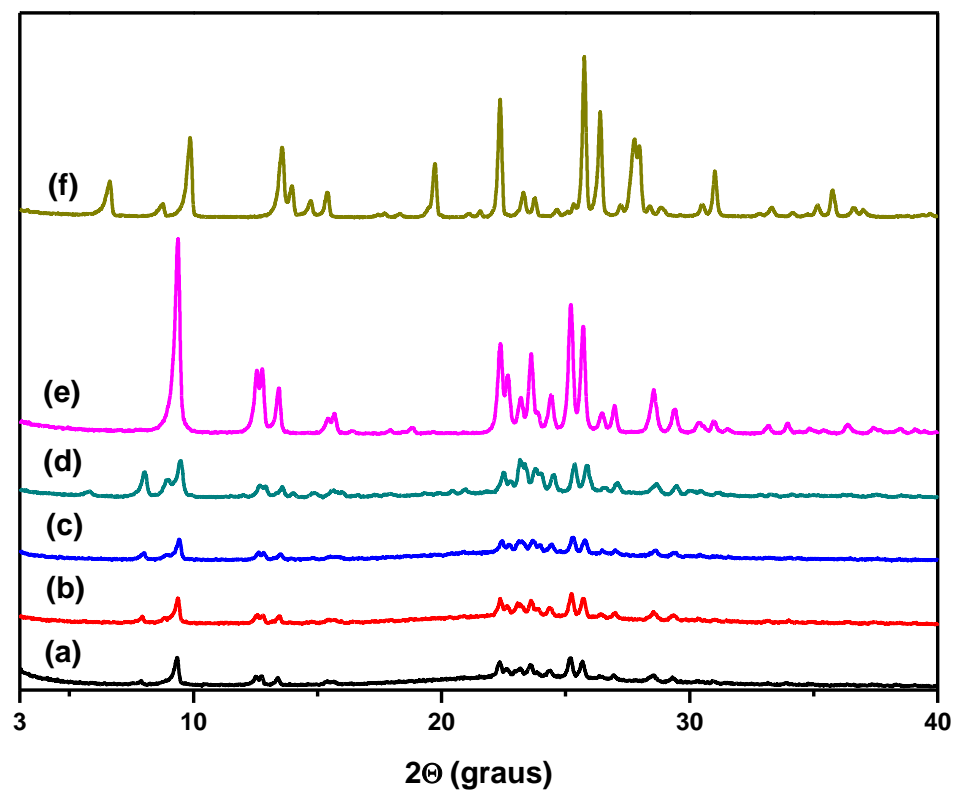

Figura. 2: Difratograma das amostras sintetizadas com $\mathrm{SiO}_{2} / \mathrm{Al}_{2} \mathrm{O}_{3}=60, \mathrm{OH} / \mathrm{SiO}_{2}=0,3$ e adição de semente de FER. (a) M60B30SF-4H; (b) M60B30SF-8H; (c) M60B30SF-12H; (d) M60B30SF-24H; (e) FER-COM; (f) Semente-MOR.

As amostras com razão $\mathrm{SiO}_{2} / \mathrm{Al}_{2} \mathrm{O}_{3}=60$, semente de $\mathrm{MOR}$ e razões $\mathrm{OH} / \mathrm{SiO}_{2}=0,2$ e 0,3 , apresentaram picos característicos da fase mordenita, mas com baixa intensidade, havendo um incremento da cristalinidade quando se elevou a razão $\mathrm{OH} / \mathrm{SiO}_{2}$ para 0,4 , mostrados na Figura 3. A Tabela 2 apresenta os dados das amostras sintetizadas utilizando sementes de MOR. 


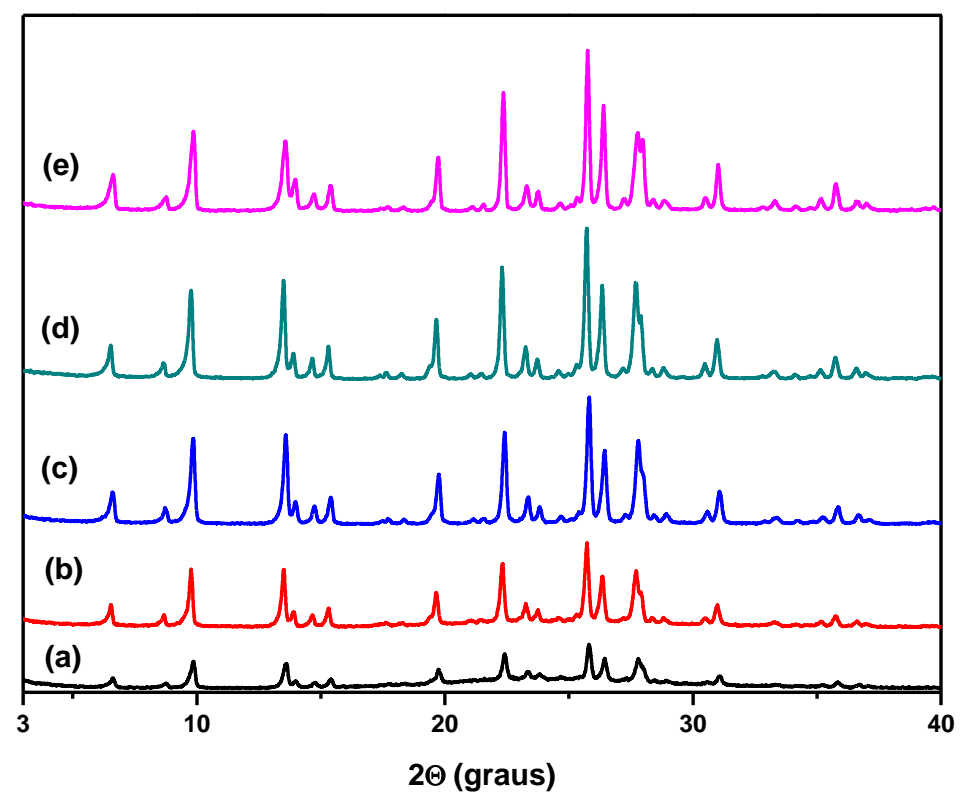

Figura 3:Difratograma das amostras com sementes de $\mathrm{MOR}$, razão $\mathrm{SiO}_{2} / \mathrm{Al}_{2} \mathrm{O}_{3}=60$ e $\mathrm{OH} / \mathrm{SiO}_{2}=0,4$. (a) M60B40SM-3H, (b) M60B40SM-6H, (c) M60B40SM-12H, (d) M60B40SM-24H;

(e) Semente-MOR.

Tabela 2: Dados das amostras com razão $\mathrm{SiO}_{2} / \mathrm{Al}_{2} \mathrm{O}_{3}=60$.

\begin{tabular}{lcccc}
\hline \multicolumn{1}{c}{ Amostra } & $\begin{array}{c}\text { Razão } \\
\text { OH/SiO }\end{array}$ & Tipo de semente & $\begin{array}{c}\text { Tempo } \\
\text { (horas) }\end{array}$ & Cristalinidade (\%) \\
\hline Semente-MOR & 0,4 & - & 24 & $100^{*}$ \\
M60B20SM_4H & 0,2 & MOR & 4 & 40 \\
M60B20SM_8H & 0,2 & MOR & 8 & 35 \\
M60B20SM_12H & 0,2 & MOR & 12 & 39 \\
M60B20SM_24H & 0,2 & MOR & 24 & 36 \\
M60B30SM_4H & 0,3 & MOR & 4 & 29 \\
M60B30SM_8H & 0,3 & MOR & 8 & 35 \\
M60B30SM_12H & 0,3 & MOR & 12 & 29 \\
M60B30SM_24H & 0,3 & MOR & 24 & 49 \\
M60B40SM_3H & 0,4 & MOR & 3 & 61 \\
M60B40SM_6H & 0,4 & MOR & 6 & 88 \\
M60B40SM_12H & 0,4 & MOR & 12 & 95 \\
M60B40SM_24H & 0,4 & MOR & 24 & \\
\hline
\end{tabular}

*Amostra padrão

Nas amostras com razão $\mathrm{SiO}_{2} / \mathrm{Al}_{2} \mathrm{O}_{3}=100$, sem sementes e razões $\mathrm{OH} / \mathrm{SiO} 2=0,2$ e 0,3 , houve a partir 48 horas o início da cristalização de uma fase, e quando comparados a carta de referência PDF 421350 [15], foi verificado se tratar da fase lamelar magadiita. A Figura 4 apresenta a evolução da cristalização das amostras com razão $\mathrm{OH} / \mathrm{SiO}_{2}=0,3$. 


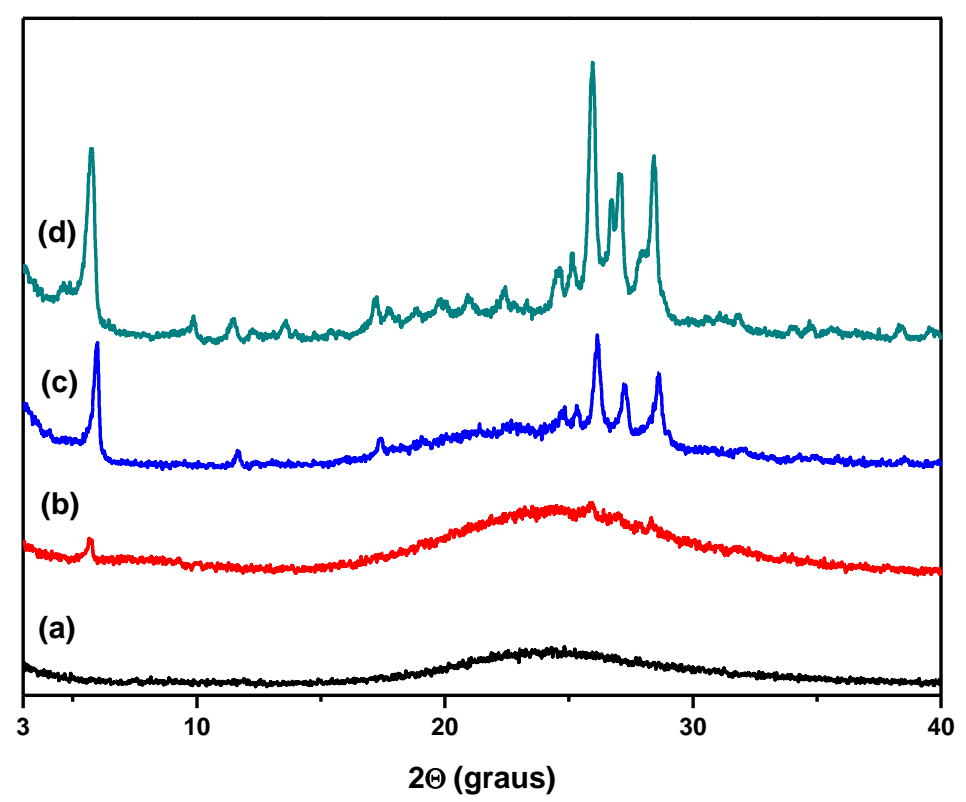

Figura 4: Difratograma das amostras com razão $\mathrm{SiO}_{2} / \mathrm{Al}_{2} \mathrm{O}_{3}=100$, sem sementes e $\mathrm{OH} / \mathrm{SiO}_{2}=0,3$. (a) M100B30-12H; (b) M100B30-24H; (c) M100B30-48H; (d) M100B30-72H.

Os resultados obtidos para as amostras com razão $\mathrm{SiO}_{2} / \mathrm{Al}_{2} \mathrm{O}_{3}$, utilizando sementes de FER e MOR, em todas as razões de $\mathrm{OH} / \mathrm{SiO}_{2}$, foram obtidas misturas binárias magadiita/ferrierita e magadiita/mordenita, indicando uma ineficácia das sementes em promover a cristalização da fase mordenita ausente de contaminantes, tão como uma preponderância desta faixa de razão $\mathrm{SiO}_{2} / \mathrm{Al}_{2} \mathrm{O}_{3}$ na obtenção da fase lamelar magadiita.

A análise dos difratogramas sobrepostos das amostras sintetizadas com adição de sementes, indicou a presença de picos característicos de ambas as fases ferrierita e mordenita. Com base nesta identificação, a quantificação de cada fase presente no material foi obtida através de metodologia similar à adotada por SILVA e colaboradores [16]. Os resultados para a quantificação do percentual de fases estão descritos na Tabela 3.

Tabela 3: Análise quantitativa percentual das fases MOR e FER nas amostras com misturas binárias.

\begin{tabular}{lcccccc}
\hline \multirow{2}{c}{ Amostra } & $\begin{array}{c}\text { Tipo de } \\
\text { semente }\end{array}$ & $\begin{array}{c}\text { Razão } \\
\mathbf{S i O}_{2} / \mathbf{A l 2 O}_{3}\end{array}$ & $\begin{array}{c}\text { Razão } \\
\mathbf{O H / S i O} \mathbf{O}_{2}\end{array}$ & $\begin{array}{c}\text { MOR } \\
(\boldsymbol{\%})\end{array}$ & $\begin{array}{c}\text { FER } \\
(\boldsymbol{\%})\end{array}$ & $\begin{array}{c}\text { Amorfo } \\
(\boldsymbol{\%})\end{array}$ \\
& & & & & & \\
\hline M20B20SF_4H & FER & 20 & 0,2 & 7 & 15 & 78 \\
M20B20SF_8H & FER & 20 & 0,2 & 7 & 44 & 49 \\
M20B20SF_12H & FER & 20 & 0,2 & 42 & 46 & 12 \\
M20B20SF_24H & FER & 20 & 0,2 & 84 & 0 & 16 \\
M20B30SF_4H & FER & 20 & 0,3 & 16 & 59 & 25 \\
M20B30SF_8H & FER & 20 & 0,3 & 78 & 22 & 0,0 \\
M20B40SF_5H & FER & 20 & 0,4 & 43 & 57 & 0,0 \\
\hline
\end{tabular}

*Todas as estimativas contêm um erro de $\pm 5 \%$ 


\subsection{ANÁLISES TÉRMICAS (ATG)}

A Figura 5 mostra as curvas termogravimétricas e suas derivadas das amostras da Semente-MOR (perda total de 13,1\%) e M20B40SF-24H (perda total de 14\%), respectivamente. O comportamento de ambos os materiais foi bastante similar, apresentando três eventos de perdas de massa, que foram atribuídos a desidratação gradual da zeólita, referente às diferentes posições das moléculas de água interagindo com a estrutura cristalina da zeólita. Os seguintes eventos consistem: (I) liberação das moléculas de água coordenadas com os cátions $\mathrm{Na}^{+}$presentes nos poros de maior dimensão da zeólita, (II) liberação do resquício da água coordenada com os cátions $\mathrm{Na}^{+}$que se encontram nos poros de menor dimensão, (III) perda de água estrutural por condensação de grupos silanol [6].
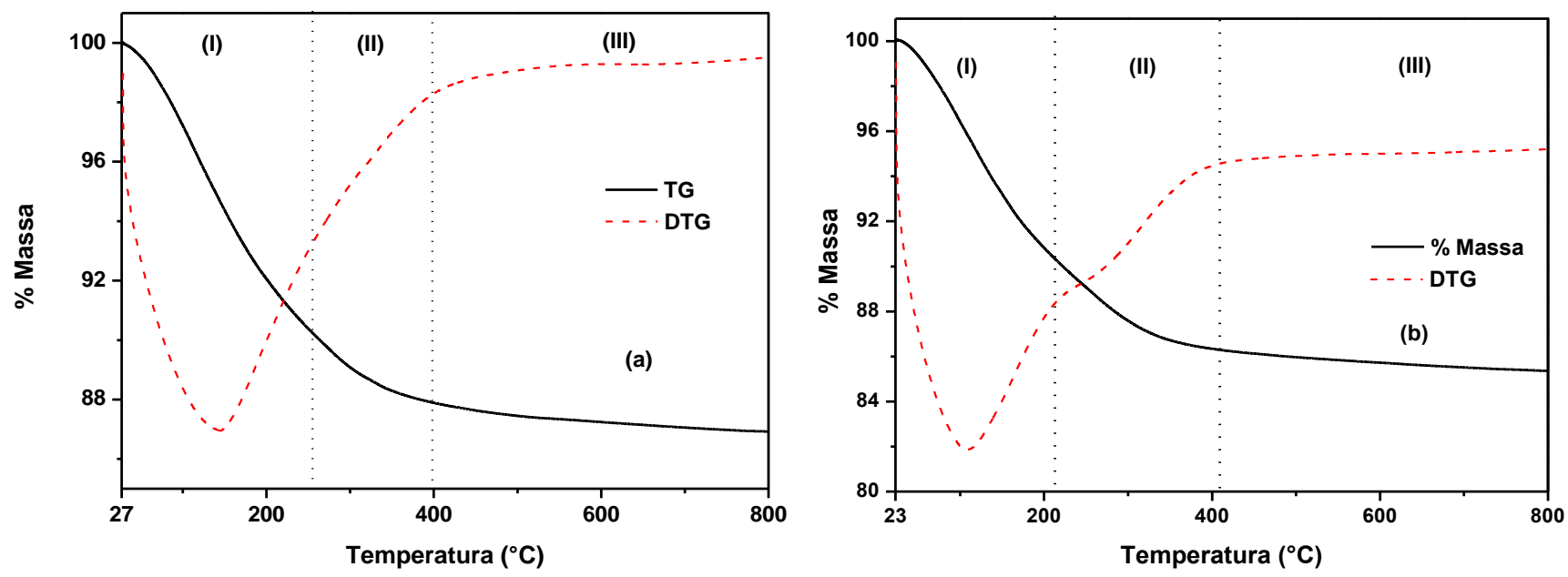

Figura 5: Análises térmicas das amostras de mordenita. (a) Semente-MOR; (b) M20B40SF-24H.

\subsection{ADSORÇÃO DE $\mathrm{N}_{2} \mathrm{~A}-196^{\circ} \mathrm{C}(\mathrm{BET})$}

As isotermas de adsorção de nitrogênio das amostras são mostradas na Figura 6, onde as isotermas representadas nas figuras são do tipo I, característica de sólidos microporosos. Os resultados de BET obtidos se apresentam muito próximos aos relatados na literatura [17], e estão organizados na Tabela 4.

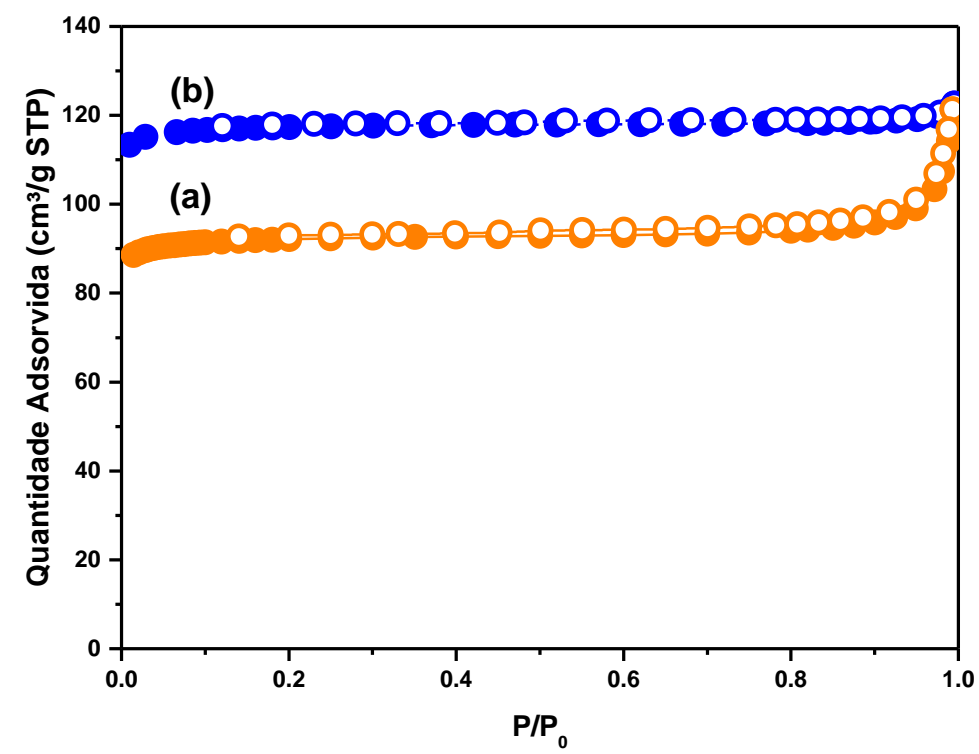

Figura 6: Isoterma de adsorção:(a) M20B40SF-24H, (b) Semente-MOR. 
Tabela 4:.Resultados das análises de adsorção de $N_{2}$ a $-196^{\circ} \mathrm{C}$.

\begin{tabular}{cccccc}
\hline Amostra & $\begin{array}{c}\text { Área } \\
\text { externa } \\
\left(\mathbf{m}^{2} / \mathbf{g}\right)\end{array}$ & $\begin{array}{c}\text { Área } \\
\mathbf{m i c r o p o r o s a} \\
\left(\mathbf{m}^{2} / \mathbf{g}\right)\end{array}$ & $\begin{array}{c}\text { Área } \\
\text { superficial } \\
\left(\mathbf{m}^{2} / \mathbf{g}\right)\end{array}$ & $\begin{array}{c}\text { Volume de poros } \\
\left(\mathbf{c m}^{3} / \mathbf{g}\right)\end{array}$ & $\begin{array}{c}\text { Volume total } \\
\left(\mathbf{c m}^{3} / \mathbf{g}\right)\end{array}$ \\
\hline Semente-MOR & 14 & 339 & 353 & 0,14 & 0,16 \\
M20B40SF-24H & 14 & 375 & 389 & 0,18 & 0,19 \\
\hline
\end{tabular}

\subsection{DESSORÇÃO À TEMPERATURA PROGRAMADA DE AMÔNIA (DTP-NH $\left.{ }_{3}\right)$}

A Figura 7 mostra as curvas de DTP-NH $\mathrm{NH}_{3}$ das amostras HSemente-MOR e HM20B40SF-24H. Observa-se a presença de dois picos, um intenso na faixa de temperatura de 150 a $350{ }^{\circ} \mathrm{C}$, e outro de baixa intensidade entre 450 e $750{ }^{\circ} \mathrm{C}$, aproximadamente. O primeiro pico está na faixa e temperatura associada à dessorção de amônia proveniente de sítios ácidos de Brønsted fracos $(\equiv \mathrm{Si}(\mathrm{OH}) \mathrm{Al} \equiv)$ e/ou de sítios ácidos de Lewis fracos $\left(\equiv \mathrm{Si}-\mathrm{O}-\mathrm{Al} \equiv\right.$, íons $\left.\mathrm{Na}^{+}\right)$. O segundo pico de dessorção está numa faixa de temperatura que caracteriza a dessorção de amônia principalmente em sítios ácidos com acidez moderada, e em alguns casos, em sítios ácidos de Brønsted fortes [18]. A acidez total (sítios ácidos de Brønsted e Lewis), em termos de concentração de sítios ácidos e a força destes, varia com a razão $\mathrm{SiO}_{2} / \mathrm{Al}_{2} \mathrm{O}_{3}$ de tal forma que se espera que a densidade de sítios ácidos decresça com o aumento da SAR.
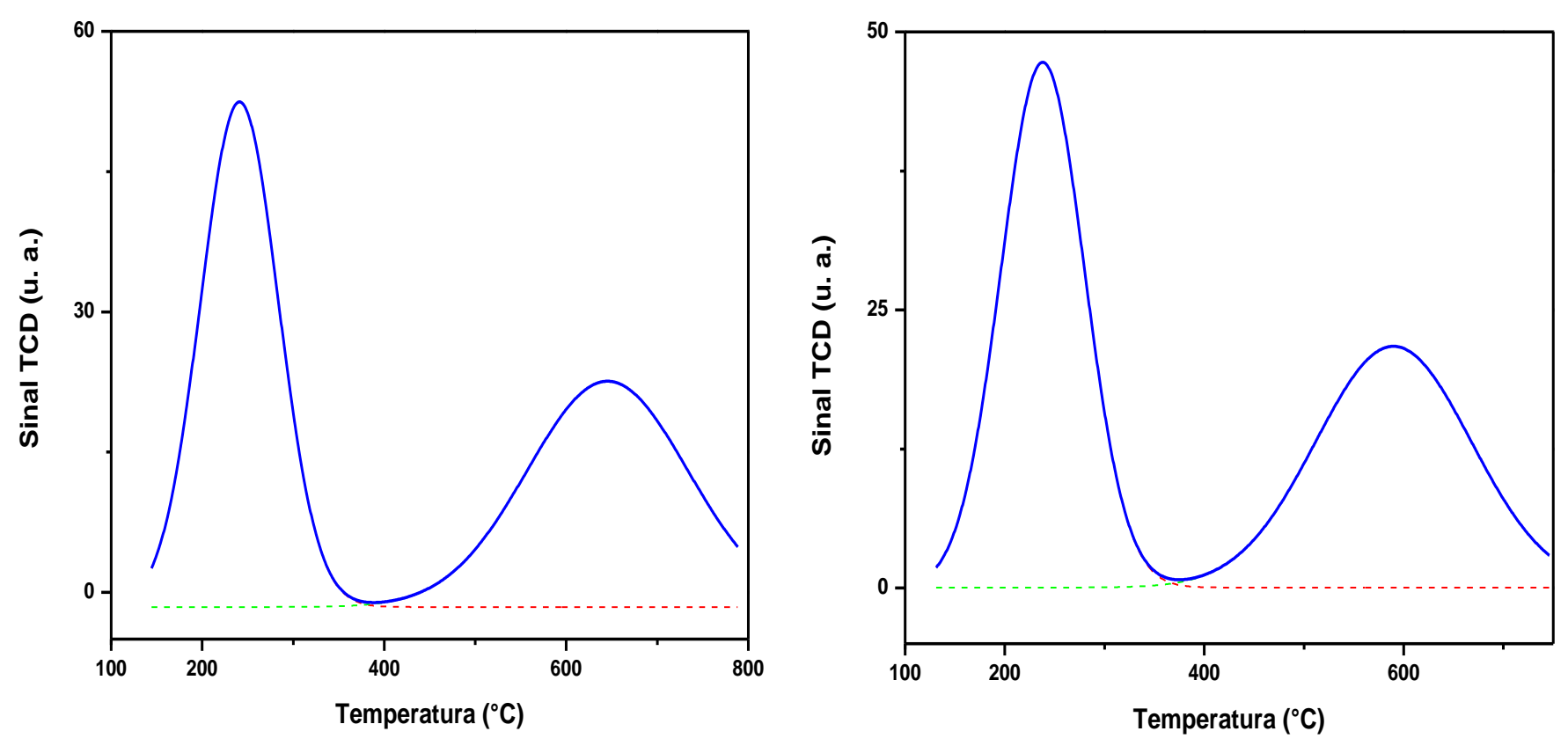

Figura 7: Curvas de TPD-NH $\mathrm{H}_{3}$ das amostras de mordenita. (a) HSemente-MOR; (b) M2OB4OSF-24H.

\subsection{MICROSCOPIA ELETRÔNICA DE VARREDURA (MEV)}

A Figura 8 representa as micrografias realizadas nas amostras Semente-MOR e M20B40SF$24 \mathrm{H}$, onde se verificou a existência de diferentes morfologias entre as amostras. A amostra Semente-MOR (a e b) apresenta aglomerados de faces cristalinas características da zeólita mordenita, com tamanho variando entre $2-5 \mu \mathrm{m}$, em contrapartida a amostra M20B40SF-24H apresenta aglomerados com formato de prismas, com a mesma variação de tamanho. De acordo com Zhang e colaboradores [19], a utilização de sementes no processo de síntese e a razão $\mathrm{SiO}_{2} / \mathrm{Al}_{2} \mathrm{O}_{3}$ inicial possuem influência direta na morfologia dos cristais, como também a uniformidade do sistema de síntese pode ter efeito na velocidade de crescimento do cristal em diferentes direções. 

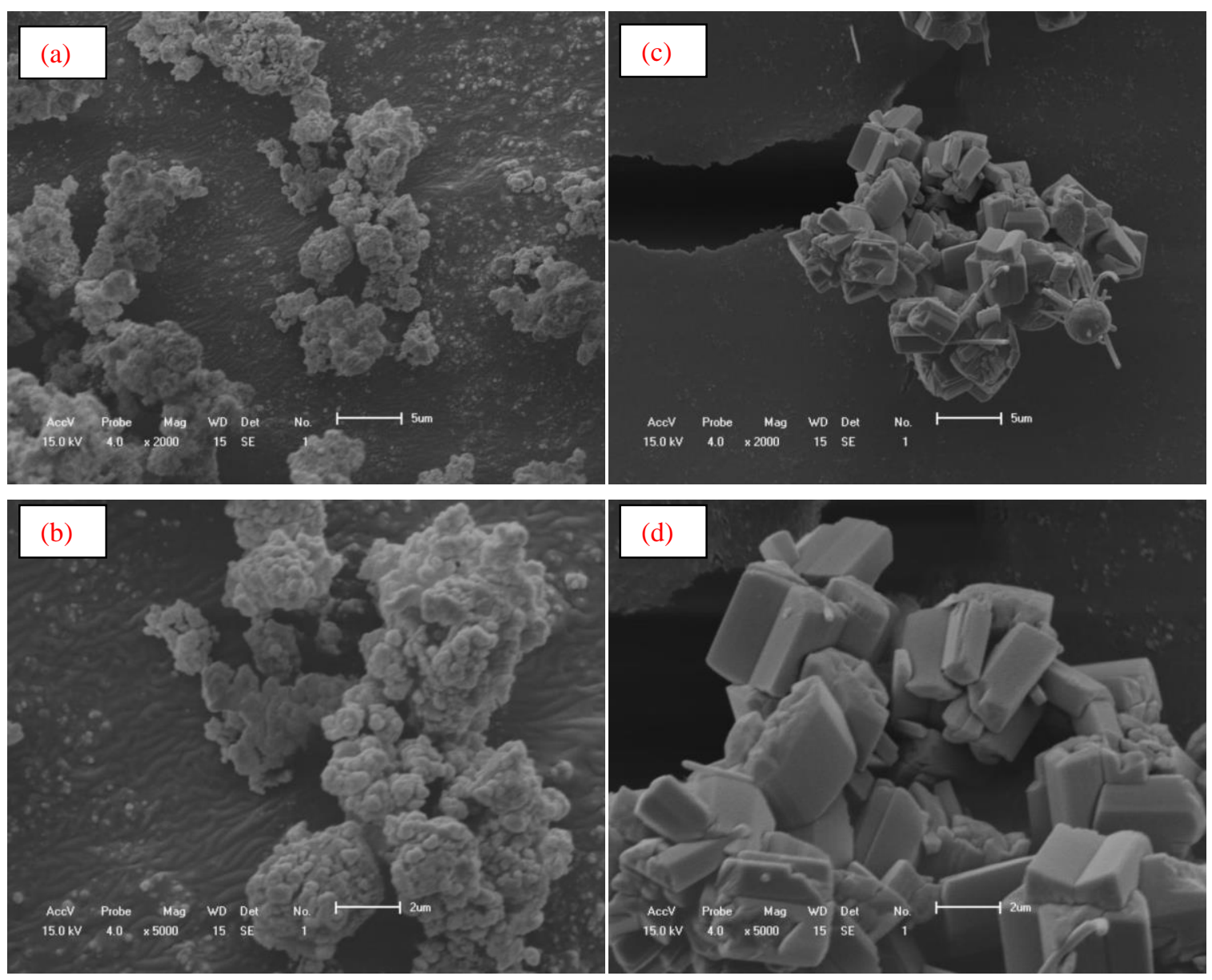

Figura 8: Micrografias das amostras de mordenita. (a) Semente-MOR com ampliação de 2.000 vezes; b) Semente-MOR com ampliação de 5.000 vezes; (c) M20B40FC-24H com ampliação de 2.000 vezes; d) M20B40FC-24H com ampliação de 5.000 vezes.

\section{CONCLUSÃO}

Nas amostras sintetizadas a partir de misturas reacionais com razão $\mathrm{SiO}_{2} / \mathrm{Al}_{2} \mathrm{O}_{3}=20$ e sem adição de sementes, quando a razão $\mathrm{OH} / \mathrm{SiO}_{2}$ foi 0,2 e 0,3 não houve a formação de fases cristalinas. A elevação desta razão para 0,4 produziu um efeito marcante, com a obtenção de amostras de mordenita altamente cristalinas, evidenciando o papel determinante da alcalinidade do meio reacional na síntese da zeólita mordenita. Nos experimentos realizados com adição de semente de FER com razão $\mathrm{OH} / \mathrm{SiO}_{2}=0,2$, foram obtidas amostras com a coexistência das fases mordenita e ferrierita. Similarmente as sínteses sem as sementes, o aumento da basicidade da mistura reacional para 0,3 e 04, favorecem a formação da fase MOR, com as amostras mais cristalinas sendo obtidas em condições mais alcalinas ( $\mathrm{razão} \mathrm{OH} / \mathrm{SiO}_{2}=0,4$ ). Enquanto que a adição de sementes de MOR torna o sistema reacional muito mais favorável a cristalização da zeólita mordenita, uma que vez, que esta fase foi obtida nos três níveis de basicidade estudados (razões $\mathrm{OH} / \mathrm{SiO}_{2}=0,2 ; 0,3$ e 0,4). Analogamente aos outros experimentos, a tendência de se obter materiais mais cristalinos em meio mais alcalino, também foi constatada com adição de sementes de MOR.

Nas sínteses com razão $\mathrm{SiO}_{2} / \mathrm{Al}_{2} \mathrm{O}_{3}=60$, sem adição de sementes, não resultou na formação de fases cristalinas, independentemente da alcalinidade do meio reacional. Isto indica que o sistema é pouco reativo, e não produz qualquer fase cristalinas em tempos de síntese curtos. Para níveis de basicidade avaliados no trabalho, a adição de sementes de FER, induziram nos tempos iniciais a formação da ferrierita, mas em tempos maiores foram obtidas amostras binárias das fases mordenita e ferrierita. No sistema com adição de sementes de $\mathrm{MOR}$ e razões $\mathrm{OH} / \mathrm{SiO}_{2}=0,2$ e 0,3 , não ocorreu a formação de fases cristalinas, porém o incremento da basicidade para 0,4 ocasionou a formação da zeólita mordenita com alta cristalinidade. 
Nas amostras com razão $\mathrm{SiO}_{2} / \mathrm{Al}_{2} \mathrm{O}_{3}=100$ e razões de $\mathrm{OH} / \mathrm{SiO}_{2}=0,2$ e 0,3 , sem adição de sementes, nos tempos iniciais foram obtidos sólidos amorfos, que foram sendo convertidos a fase lamelar magadiita à medida que o tempo de cristalização aumentou. A elevação da basicidade para 0,4 , produziu apenas materiais amorfos em todos os tempos de cristalização. De forma similar, quando se adicionou semente de FER e em todas as razões de $\mathrm{OH} / \mathrm{SiO}_{2}$, os materiais inicialmente apresentaram picos característicos de ferrierita relacionados a própria semente, e gradativamente foram sendo convertidos na fase magadiita. Nos experimentos com adição de sementes de MOR, não houve uma evolução da cristalinidade do material com as razões $\mathrm{OH} / \mathrm{SiO}_{2}=0,2$ e 0,3 , onde com a basicidade igual a 0,2 o sólido final obtido já estaria se convertendo para a fase magadiita. Com o incremento da razão $\mathrm{OH} / \mathrm{SiO}_{2}$ para 0,4 , houve um aumento da cristalinidade do material em comparação ao material obtido com as outras duas faixas de basicidade, obtendo-se a mordenita com baixa cristalinidade nos tempos iniciais, posteriormente com o tempo de 24 horas se obteve a fase magadiita.

\section{AGRADECIMENTOS}

A PETROBRAS, ANP e FINEP pelo apoio financeiro. A CAPES e ao CNPQ pelas bolsas concedidas.

\section{REFERÊNCIAS}

1. Shiokawa K, Ito M, Itabashi K. Crystal structure of synthetic mordenites. Zeolites. 1989 Maio: 9 (3):170176, doi:10.1016/0144-2449(89)90021-3.

2. Haimidi F, Dutartre R, Direnzo F, Bengueddach A, Fajula F. Proceedings of the 12th International Zeolite Conference 3 (1999) 1803.

3. Meier WM, Z. The crystal structure of mordenite. Kristallogr. 1961Out: 115 (5-6) 439-450, doi: 10.1524/zkri.1961.115.5-6.439.

4. Fernandes LD, Monteiro JLF, Sousa-Aguiar EF, Martinez A, Corma A. Ethylbenzene hydroisomerization over bifunctional zeolite based catalysts: The influence of framework and extraframework composition and zeolite structure. Journal of Catalysis. 1998 Abr: 177 (2): 363-377, doi:10.1006/jcat.1998.2111.

5. Li G, Hou H, Lin R. Rapid synthesis of mordenite crystals by microwave heating. Solid State Sciences. 2011 Jan: 13 (3) 662 - 664, doi: 10.1016/j.solidstatesciences.2010.12.040.

6. Aly HM, Moustafa ME, Abdelrahman EA. Synthesis of mordenite zeolite in absence of organic template. Advanced Powder Technology. 2012 Out: 23 (6) 757-760, doi: 10.1016/j.apt.2011.10.003.

7. Beyer HK, Belenykaja IM, Mishin IW, Borbely G, in: P.A.Jacobs, N.I. Jaeger, P. Jiru, V.B.Kazansky, G. Schulz-Ekloff (Eds.), Structure and Reactivity of Modified Zeolites, Elsevier,Amsterdam, 1984, p. 133.

8. Jongkind H, Datema KP, Nabuurs S, Seive A, Stork WHJ. Synthesis and characterisation of zeolites using saturated cyclic amines as structure-directing agents, Microporous Materials. 1997 Jul: 10 (4-6) 149-161, doi:10.1016/S0927-6513(97)00005-9.

9. Shao C, Kim HY, Li X, Park S.J, Lee DR. Synthesis of high-silica-content mordenite with different $\mathrm{SiO}_{2} / \mathrm{Al}_{2} \mathrm{O}_{3}$ ratios by using benzene-1,2-diol as additives, Materials Letters. 2002 Set: 56 (1-2) 24-29, doi:10.1016/S0167-577X(02)00411-1.

10. Qian B, Guo G, Wang X, Zeng Y, Sun Y, Long Y. Templating of high-silica zeolites by tetrahydrofuran in the Na system, Phys. Chem. Chem. Phys. 2001 Ago: 3 (5) 4164-4169, doi: 10.1039/B103230P.

11. CASCI JL. Zeolite molecular sieves: preparation and scale-up, Microporous and Mesoporous Materials. 2005 Ago: 3 (82) 217-226, doi:10.1016/j.micromeso.2005.01.035.

12. Kerr GT. Chemistry of crystalline aluminosilicates. IV. Factors affecting the formation of zeolites $X$ and B, J. Phys. Chem. 1968 Abr: 72 (4) 1385-1386, doi: 10.1021/j100850a056.

13. Petitti DB, Crooks VC, Buckwalter JG, Chiu V. Blood pressure levels before dementia, Arch Neurol. 2005 Jan: 62 (1) 112-116, doi:10.1001/archneur.62.1.112.

14. Meneton P, Jeunemaitre X, de Wardener HE, MacGregor GA. Links between dietary salt intake, renal salt handling, blood pressure, and cardiovascular diseases. Physiol Rev. 2005 Apr: 85 (2) 679-715, doi:10.1152/physrev.00056.2003.

15. Jenkins PF. Making sense of the chest x-ray: a hands-on guide. New York: Oxford University Press; 2005. 194 p. 
16. Riffenburgh RH. Statistics in medicine. 2nd ed. Amsterdam (Netherlands): Elsevier Academic Press; 2006. Chapter 24, Regression and correlation methods; p. 447-86, doi: 10.1016/B978-0-12-3848642.00025-1

17. Zhao C. Development of nanoelectrospray and application to protein research and drug discovery [dissertation]. Buffalo (NY): State University of New York at Buffalo; 2005. 276 p.

18. Rice AS, Farquhar-Smith WP, Bridges D, Brooks JW. Canabinoids and pain. In: Dostorovsky JO, Carr DB, Koltzenburg M, editors. Proceedings of the 10th World Congress on Pain; 2002 Aug 17-22; San Diego, CA. Seattle (WA): IASP Press; c2003. p. 437-68. 